\title{
Limited validity of the Kubo formula for thermal conduction in modular quantum systems
}

\author{
Jochen Gemmer and Robin Steinigeweg

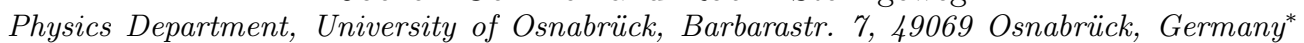 \\ Mathias Michel \\ Institute of Theoretical Physics I, University of Stuttgart, Pfaffenwaldring 57, 70550 Stuttgart, Germany
}

(Dated: April 21, 2018)

\begin{abstract}
The Kubo formula describes a current as a response to an external field. In the case of heat conduction there is no such external field. We analyze why and to what extend it is nevertheless justified to describe heat conduction in modular quantum systems by the Kubo formula. "Modular" we call systems that may be described as consisting of weakly coupled identical subsystems. We explain in what sense this description applies to a large class of systems. Furthermore, we numerically evaluate the Kubo formula for some finite modular systems. We compare the results with data obtained from the direct numerical solution of the corresponding time-dependent Schrödinger equation.
\end{abstract}

PACS numbers: 05.60.Gg, 44.10.+i, 05.70.Ln

\section{INTRODUCTION}

There are essentially two reasons that can cause a current of particles like, e.g., electrons through solids: Either there is an electric field dragging the electrons in some direction or the electron density is spatially non-uniform, i.e., there is a density gradient causing the electrons to diffuse. For systems featuring normal transport the currents in those two cases are supposed to be determined by

$$
\begin{gathered}
\boldsymbol{j}=L_{F} \boldsymbol{F}, \\
\boldsymbol{j}=-L_{D} \nabla \rho,
\end{gathered}
$$

where $\boldsymbol{j}$ is the current, $\boldsymbol{F}$ the external force, $\rho$ the spatial density and the $L$ 's are the pertinent response coefficients. As will be briefly outlined below the derivation of (1a) from the corresponding scenario is rather straightforward and leads to the Kubo formula 1.2 (KF). A direct derivation of (1b) from its underlying scenario seems to be significantly more subtle. This is a crucial point for the analysis of heat conduction since, although being rather similar to the above case otherwise, the scenario corresponding to (1a) does not exist for heat transport. There simply is no external field which could exert a force on heat, heat is always driven by an energy density (temperature) gradient as described by (1b). Nevertheless, as will also be sketched below, there have been attempts to apply the derivation of (1a) to thermal conduction which eventually implies the application of the KF also in this case 1.2 .3 .4 .

Let us briefly recall the derivation of the KF. An external field gives rise to an additional energy $H^{\prime}=\int \rho U \mathrm{~d} V$ with $-\nabla U=\boldsymbol{F}$. This term is routinely treated as a perturbing addend to the Hamiltonian of the system yielding an expression for the induced current. In this expression
$\hat{H}^{\prime}$ itself no longer appears only its derivative with respect to time which in the case of a (spatially constant) external field reads $\dot{H}^{\prime}=-\boldsymbol{j} \boldsymbol{F}$. Thus one obtains a relation of the form $(1 \mathrm{a}) . L_{\boldsymbol{F}}$ is given by the Kubo formula and reads

$$
L(\omega)=\frac{1}{V} \int_{0}^{\infty} \mathrm{d} t \mathrm{e}^{-\mathrm{i} \omega t} \int_{0}^{\beta} \mathrm{d} \tau \operatorname{Tr}\left\{\hat{\rho}_{0} \hat{j}(0) \hat{j}(t+\mathrm{i} \tau)\right\}
$$

where $\hat{\rho}_{0}$ is the Gibbsian equilibrium state, $V$ the volume and $L(\omega)$ is the response coefficient which describes the conductivity at frequency $\omega$.

In the case of heat conduction no additional energy arises from the internal force (temperature gradient) and thus the stimulus of the current cannot be incorporated into the Hamiltonian. Hence the above line of reason does not apply. To nevertheless justify a Kubo-type formula for thermal conductivity basically two types of arguments are brought forth:

i) The hypothesis of local equilibrium

The state that one gets by boldly writing down a Gibbs state with a spatially non-uniform temperature that varies little $(\Delta T(\boldsymbol{x}))$ around some mean $\beta_{0}=1 / T_{0}$ reads

$$
\hat{\rho}_{\text {leq }}=Z^{-1} \exp \left(-\beta_{0} \int \mathrm{d} \boldsymbol{x} \frac{1-\Delta T(\boldsymbol{x})}{T_{0}} \hat{h}(\boldsymbol{x})\right)
$$

( $Z$ being the partition function, $\hat{h}(\boldsymbol{x})$ the local energy density and $T_{0}$ respectively $\beta_{0}$ some mean temperature) and is called a local equilibrium state ( $\left.\operatorname{cf}^{\text {Ref }}{ }^{5.6}\right)$. Its physical significance is somewhat vague since it is not a real equilibrium state. However, adding a "pseudoperturbation" of the form $\hat{H}_{\mathrm{ps}}^{\prime}=-\int \mathrm{d} \boldsymbol{x} \Delta T(\boldsymbol{x}) \hat{h}(\boldsymbol{x}) / T_{0}$ to the system's Hamiltonian and calculating the Gibbs state at uniform $\beta_{0}$ of the perturbed system formally yields the above local equilibrium state (3). Thus this term is said to somehow model the effect of the internal temperature gradient, although the local equilibrium 
state features no current. Nevertheless, proceeding like described above and taking $\hat{H}_{\mathrm{ps}}^{\prime}$ for an external perturbation yields a transport coefficient as given by (2) (just multiplied by $\beta_{0}$ ) with a thermal current $\hat{j}$ defined by $\dot{h}+\nabla j=0$.

ii) The entropy production argument

If, e.g., an electrical current in some conducting solid runs along an electric field, potential energy arising from this field, $H^{\prime}$, is converted to heat, $Q$. Thus one has $-\dot{H}^{\prime}=\dot{Q}$. Hence the entropy production is $\dot{S} \geq \dot{Q} / T$. On the other hand entropy production is assumed to be of the form $\dot{S}=\boldsymbol{J} \cdot \nabla T / T^{2}$ (see Refs, ${ }^{7.8 .9 .10}$ ). Combining these equations and boldly replacing the " $\geq$ " by an "=" yields $\dot{H}^{\prime}=-\boldsymbol{J} \cdot \nabla T / T$. This can formally be incorporated in the derivation of the KF outlined above and yields the same result as the hypothesis of local equilibrium. The crucial shortcoming of this argument is that if heat flows along a temperature gradient, obviously no heat is produced, only entropy. Thus, in this case one would definitely have to consider a ">" and then this argument yields no concise result.

Thus it has often been pointed out that those concepts do not provide a rigorous justification of the KF for thermal conduction ${ }^{5,11,12,13,14,15,16}$ which remains an open question. Furthermore, the KF has been counterchecked only for few concrete systems, see Refs ${ }^{17.18}$. Despite those conceptual problems the application of the KF to heat conduction is today a standard technique ${ }^{19.20 .21}$. Thus the main intend of the paper at hand is to give a derivation of a Kubo-type formula for thermal conduction which is more convincing, thereby also pointing out the limits of its validity.

\section{KUBO FORMULA FOR FINITE QUANTUM SYSTEMS}

Before we proceed with our main line of thought in Sect. III we shortly comment on another difficulty that arises if one wants to compute the transport coefficients of finite quantum systems from the KF. This point deserves consideration, since, in general, an infinite system cannot be treated numerically, thus in many cases a transport coefficient of an (periodic) infinite system can only be computed from analyzing a finite piece of the infinite system (cf. Ref ${ }^{22}$ ).

Of course, a key question is whether or not the transport is normal at all. For infinite systems the coefficient is believed to take the form $L(\omega)=D_{d r} \delta(0)+\kappa(\omega)$. $D_{d r}$ is called the Drude weight and whenever it is nonzero for infinite systems the transport is assumed to be ballistic. If it is zero, the normal (diffusive) conductivity is supposed to be given by $\kappa(0), \kappa(\omega)$ being a smooth function without any singularities 20.22 . However, this distinction is problematic if the $\mathrm{KF}$ is evaluated on the basis of a finite quantum system. In this case $L(\omega)$ consists of a sum of delta peaks at different frequencies without any non-singular contribution ${ }^{20,22}$. So, technically speaking, one cannot find normal transport in a finite quantum system, it is either ballistic or none. There are different ideas on how the transition to infinite systems could produce normal transport. Sometimes the singularity at $\omega=0$ is assumed to broaden ("imaginary broadening") but maintain its weight such that $L(0)=D_{d r} / \tau$ is nonsingular, where $\tau$ is some inverse width of the Drude-peak which is hard to evaluate from a closed finite system ${ }^{22}$. In other approaches $L(\omega)$ is averaged over small frequency intervals $\delta \omega$ and the resulting smooth function is extrapolated down to $\omega=0$ in order to determine the normal conductivity 22.23 . The result depends, of course, on how $\delta \omega$ is chosen. In general it appears to be difficult to determine transport type and conductivity merely from the Hamiltonian of a finite piece of an infinite system without any further assumptions.

Thus, except for demonstrating the limited validity of the KF, the paper at hand also suggests a consistent method to infer the heat conduction behavior of an infinite system from analyzing an adequate finite piece of it.

\section{MODULAR QUANTUM SYSTEMS AND DIFFUSIVE DYNAMICS}

We consider systems consisting of identical many-level subunits which are weakly coupled by identical nextneighbor interactions. We call those systems "modular". For simplicity, we analyze chains and rings of that kind. Their Hamiltonians may be denoted as

$$
\hat{H}=\sum_{\mu=1}^{N} \hat{h}(\mu)+\sum_{\mu=1}^{N-1} \hat{V}(\mu, \mu+1),
$$

where $\hat{h}(\mu)$ is the local Hamiltonian of some subunit $\mu, N$ the total number of subunits and $\hat{V}(\mu, \mu+1)$ represents a next-neighbor interaction between the subsystems $\mu$ and $\mu+1$ (Schematic examples of such modular systems are depicted in Fig. 10and Fig. 8). The one-dimensional character is not crucial here. Everything derived below can be generalized straightforwardly to modular systems featuring arbitrary multi-dimensional "net-structures". So what sort of physical systems are modular systems? First of all interacting nano-structures like arrays of quantum dots, etc., might fit this scheme. But modular structures may also be achieved by operationally coarse graining periodic (or also slightly disordered) systems like spin chains, crystals, etc. in modules such that each module containins many elementary cells. The interactions between the modules then represent the couplings. Since interactions are typically rather short ranged, increasing the "grain size" will eventually result in a description in which only adjacent modules are coupled. Furthermore, these next neighbor couplings will become weaker, such that they finally might be considered as weak. This "weak coupling", as well as other criteria which determine whether or not there is regular transport, will be 
given more precisely below. They may all depend on the choice of the grain size. This reflects the fact that regular thermodynamic behavior may only be expected for a spatially coarse enough description. Whether or not the criteria for regular transport are fulfilled at some grain size has to be investigated for any system individually. However, the description of a system as a modular system should be possible for very many systems. Thus, in order to keep everything as general as possible, we do not specify our systems in much more detail than already given by (46) (not even the numerical examples in Sect. VIII. But the concrete application of the results at hand to, e.g., spin chains is under way.

What does diffusive transport mean in the context of modular systems? Diffusive heat transport is defined by Fourier's law which is essentially given by (1b). For modular systems we replace the spatial gradient by the energy difference between two adjacent subunits, i.e., the current $j(\mu)$ from subunit $\mu$ to subunit $\mu+1$ should obey $j(\mu)=D(h(\mu)-h(\mu+1))$. A discrete form of the continuity eq. for modular systems reads $\dot{h}(\mu)=j(\mu-1)-j(\mu)$. Combining those eqs. yields, e.g., for a chain

$$
\begin{aligned}
\frac{\mathrm{d} h_{1}}{\mathrm{~d} t} & =D(h(2)-h(1)) \\
\frac{\mathrm{d} h_{\mu}}{\mathrm{d} t} & =D(h(\mu-1)+h(\mu+1)-2 h(\mu)), \\
\frac{\mathrm{d} h_{N}}{\mathrm{~d} t} & =D(h(N-1)-h(N)) .
\end{aligned}
$$

(This may be viewed as a discrete form of the timedependent version of Fourier's law: $\dot{h}(\boldsymbol{x})=(\kappa / c) \Delta h(\boldsymbol{x})$.) If the motion of energy through the closed system (which is entirely controlled by its Hamiltonian and the Schrödinger eq.) can be described by the above set of eqs., the thermal transport is diffusive. The conductivity is obviously related to $D$.

Our paper is roughly organized as follows: We consider the dynamics of a modular system without any external forces, starting from a local equilibrium state as given by (3). We find that, under various conditions on the model parameters, the motion of the energy can be described by (5) for a short first time-step (cf. Sect. (V), where $D$ is essentially determined by the KF. After that time-step the system is unfortunately no longer in a local equilibrium state. It can, however, be shown that almost all states sharing some crucial properties with the local equilibrium state will also give rise to energy dynamics in accord with (5.5) for another time-step. That means, fully evolved local equilibrium is dispensable. Thus one can, iterating the result for short time-steps, conclude that (5) provides a correct description for all times (cf. Sect. VI) and thus justify the application of the KF. Eventually we evaluate the KF for some concrete finite models and compare the results with the energy dynamics obtained from a direct numerical integration of the corresponding time-dependent Schrödinger eqs. (cf. Sect. VIII).

\section{DEFINITION OF LOCAL ENERGY CURRENTS IN MODULAR QUANTUM SYSTEMS}

Consider a Hamiltonian as given by (4). In the paper at hand we define the local energy operator at site $\mu$ simply as $\hat{h}(\mu)$ rather than $\hat{h}(\mu)+(\hat{V}(\mu, \mu-1)+\hat{V}(\mu, \mu+1)) / 2$. This way the sum of all local energies does not represent the total energy and is not a strictly conserved quantity. But the local energy operators are defined on strictly separated subspaces of the product space on which the full system is defined. Excluding the interactions from the local energies makes a consistent partition of the system into mutually disjoint (smallest) subunits possible, such that on each subunit a local (equilibrium) state may be defined independently. This would be impossible if one included the interactions in the local energies. However, if one wants to define an energy current based on the evolutions of those local energies, the sum of the local energies has to be at least approximately conserved, i.e., the part of the full energy associated with the local energies, $\operatorname{Tr}\{\hat{\rho} \hat{h}(\mu)\}$, has to be much bigger than the part associated with the interactions, $\operatorname{Tr}\{\hat{\rho} \hat{V}(\mu, \mu+1)\}$. This eventually means that the interactions have to be weak.

For conserved quantities the discrete continuity equation $\dot{h}(\mu)=j(\mu-1)-j(\mu)$ suggests the definition of a local current operator $\frac{15.16,22}{}$ via $(\hbar=1)$

$$
\begin{aligned}
\frac{\mathrm{d}}{\mathrm{d} t} \hat{h}(\mu) & =\mathrm{i}[\hat{H}, \hat{h}(\mu)] \\
& =\underbrace{\mathrm{i}[\hat{V}(\mu-1, \mu), \hat{h}(\mu)]}_{\hat{j}(\mu-1)}+\underbrace{\mathrm{i}[\hat{V}(\mu, \mu+1), \hat{h}(\mu)]}_{-\hat{j}(\mu)} .
\end{aligned}
$$

However, rewriting the above equation for the temporal change of $\hat{h}(\mu+1)$ produces another expression for the local current $\hat{j}(\mu)=\mathrm{i}[\hat{V}(\mu, \mu+1), \hat{h}(\mu+1)]$. This can only be consistent if

$$
[\hat{V}(\mu, \mu+1), \hat{h}(\mu)+\hat{h}(\mu+1)] \approx 0 .
$$

But this expression can be interpreted as the temporal change of the sum of two adjacent local energies, if only the interaction between the respective subunits was present. According to the weak coupling precondition, this sum of local energies is approximately conserved. Or at least its fluctuations are small compared to its mean value as long as the energy contained in the subunits is not too small. This means in particular that (7) may safely be assumed as long as the temperature is not too small (we will come back to that condition later). Thus a sort of symmetrized local current is defined by

$$
\hat{j}(\mu)=\mathrm{i}[\hat{V}(\mu, \mu+1), \hat{S}], \quad \hat{S}=\frac{1}{2}(\hat{h}(\mu+1)-\hat{h}(\mu)) .
$$

The local current operator is strictly defined within the product space spanned by the corresponding adjacent subunits. Its expectation value is determined only by the 
reduced state of those adjacent subunits. Thus, within this framework, the relation between current and temperature difference may be determined on the basis of a reduced system consisting of only two interacting subunits. In this reduced system the current is simply the temporal change of $\hat{S}$. And $\hat{S}$ may be interpreted as the operator for the skewness of the energy distribution between the two subunits.

\section{LOCAL ENERGY CURRENTS AS A RESPONSE TO LOCAL TEMPERATURE GRADIENTS}

We now investigate the relation between temperature difference $\Delta T$ and the short time behavior of the energy current $j$. Inspite of having commented in Sect. \ on the local equilibrium state in a rather critical way we now analyze the short time dynamics, i.e., the formation of a current of an initial local equilibrium state as given by (3). Since we are not deducing any pseudo potentials here, this does not imply the application of the hypothesis of local equilibrium in the sense described in Sect. II However, if one starts with a local equilibrium state and considers only a short time step, the question arises whether the system will be in (another) local equilibrium state after this time step? The answer is no, but this issue is addressed in detail in Sect. VI For the moment we thus simply consider the local equilibrium state $\hat{\rho}(T, \Delta T)$ which is defined for the two coupled subunits which form our reduced system (for simplicity, those are only labeled " 1 " and " 2 " in the following):

$$
\hat{\rho}(T, \Delta T) \approx \hat{\rho}_{0}\left(\hat{1}+\frac{\Delta T}{T^{2}} \hat{S}\right), \quad \hat{\rho}_{0}:=\frac{\exp \left(\frac{-(\hat{h}(1)+\hat{h}(2))}{T}\right)}{Z^{2}(T)} .
$$

( $Z$ is the partition function of one subsystem and we chose units of temperature and energy as $k_{\mathrm{B}}=1$, $\hbar=1$.) Here $\hat{\rho}_{0}$ is obviously a "global" equilibrium state with both subunits at the same temperature $T$. Unfortunately, the current for this state vanishes, i.e., $\operatorname{Tr}\{\hat{\rho}(T, \Delta T) \hat{j}\}=0$ (if the partial traces of $\hat{V}$ with respect to the subunits vanish, which can be demanded without loss of generality).

Thus one has to proceed in a slightly different way: We analyze an approximate short time evolution of the expectation value

$$
S(\tau)=\operatorname{Tr}\{\hat{\rho}(T, \Delta T) \hat{S}(\tau)\}
$$

and consider it's derivative with respect to time for some small but finite $\tau$. This derivative is, according to the Heisenberg equation of motion and the definition in (8), the current at time $\tau$. The time evolution of the operator $\hat{S}(\tau)$ is computed by means of a truncated Dyson series, i.e.,

$$
\begin{aligned}
& \hat{S}(\tau)=\hat{D}^{\dagger}(\tau) \hat{S} \hat{D}(\tau) \quad \text { with } \\
& \hat{D}(\tau) \approx \hat{1}-\mathrm{i} \hat{U}_{1}(\tau)-\hat{U}_{2}(\tau)
\end{aligned}
$$

and the time evolution operators (see Ref ${ }^{24,25}$ )

$$
\begin{aligned}
& \hat{U}_{1}(\tau)=\int_{0}^{\tau} \mathrm{d} \tau^{\prime} \hat{V}\left(\tau^{\prime}\right), \\
& \hat{U}_{2}(\tau)=\int_{0}^{\tau} \mathrm{d} \tau^{\prime} \int_{0}^{\tau^{\prime}} \mathrm{d} \tau^{\prime \prime} \hat{V}\left(\tau^{\prime}\right) \hat{V}\left(\tau^{\prime \prime}\right) .
\end{aligned}
$$

Here the time dependence of the $\hat{V}(\tau)$ 's is defined on the basis of an interaction picture, i.e., only generated by the local Hamiltonians $\hat{h}(1)+\hat{h}(2)$ rather than the full Hamiltonian. This yields in second order approximation

$$
\hat{S}(\tau) \approx \hat{S}+\mathrm{i}\left[\hat{U}_{1}, \hat{S}\right]+\hat{U}_{1} \hat{S} \hat{U}_{1}-\left(\hat{S} \hat{U}_{2}+\hat{U}_{2}^{\dagger} \hat{S}\right) .
$$

(For simplicity of notation, we suppress the time argument of the $\hat{U}$ 's here and in the following.) Computing $S(\tau)$ defined in (10) by plugging in the operator (14) and the state (9) yields

$$
S(\tau)=\frac{\Delta T}{T^{2}}\left(\operatorname{Tr}\left\{\hat{\rho}_{0} \hat{S}^{2}\right\}+\frac{1}{2} \operatorname{Tr}\left\{\hat{\rho}_{0}\left[\hat{U}_{1}, \hat{S}\right]^{2}\right\}\right),
$$

where we have exploited $\hat{U}_{2}+\hat{U}_{2}^{\dagger}=\hat{U}_{1}^{2}$ (which follows from the definitions (12) and (13)) and $\left[\hat{\rho}_{0}, \hat{S}\right]=0$ as well as $\left[\hat{U}_{1}, \hat{\rho}_{0}\right] \approx 0$. As already mentioned the latter is valid for high enough temperatures (remember discussion below (7)). Realizing, by using the definition of $\hat{U}_{1}$ and (8), that

$$
\left[\hat{U}_{1}, \hat{S}\right]=-\mathrm{i} \int_{0}^{\tau} \hat{j}\left(\tau^{\prime}\right) \mathrm{d} \tau^{\prime}
$$

where again $\hat{j}\left(\tau^{\prime}\right)$ is defined according to the interaction picture, one can write the derivative with respect to time of (15) as

$$
\frac{\mathrm{d}}{\mathrm{d} \tau} S(\tau)=-\frac{\Delta T}{T^{2}} \int_{0}^{\tau} \operatorname{Tr}\left\{\hat{\rho}_{0} \hat{j}\left(\tau^{\prime}\right) \hat{j}(\tau)\right\} \mathrm{d} \tau^{\prime} .
$$

Eventually, substituting $t^{\prime}=\tau-\tau^{\prime}$ and $t=\tau$

$$
\frac{\mathrm{d}}{\mathrm{d} t} S(t)=j(t)=-\frac{\Delta T}{T^{2}} \int_{0}^{t} \underbrace{\operatorname{Tr}\left\{\hat{\rho}_{0} \hat{j}(0) \hat{j}\left(t^{\prime}\right)\right\}}_{=: C\left(t^{\prime}\right)} \mathrm{d} t^{\prime},
$$

the current is essentially given by an integration over the current auto-correlation function, $C\left(t^{\prime}\right)$, just like in the KF. Let the timescale on which this correlation typically decays be $\tau_{c}$. Then, if the approximation for $S(t)$ (second order Dyson series) holds for times larger than $\tau_{c}$ (which will be analyzed below), the current will indeed assume a steady value after $\tau_{c}$ which is proportional to $\Delta T$. Thus, first of all, in the case of free heat transport without any external baths Fourier's law is confirmed for the short-time evolution of a local equilibrium state. The conductivity is now defined by

$$
\kappa=\frac{1}{T^{2}} \int_{0}^{\tau_{0}} C(t) \mathrm{d} t \quad \text { with } \quad \tau_{c}<\tau_{0}<\tau_{d}
$$


where $\tau_{d}$ is the timescale on which the second order approximation for the Dyson series brakes down $\left(\tau_{d}\right.$ will be evaluated more concretely below). Within this interval the integral should not depend much on its upper limit $\tau_{0}$. Nevertheless, for finite quantum systems (for which $C(\omega)$ is just a set of peaks) this value may differ considerably from the one which is produced by letting the upper limit go to infinity. To see this and the relation to the $\mathrm{KF}$ it is instructive to consider $C(\omega)$ and $\bar{C}(\omega)$ defined by

$$
\begin{aligned}
& C(\omega)=\int_{-\infty}^{\infty} \frac{\mathrm{e}^{-\mathrm{i} \omega t}}{2 \pi} C(t) \mathrm{d} t, \\
& \bar{C}(\omega)=\frac{1}{\delta \omega} \int_{\omega-\frac{\delta \omega}{2}}^{\omega+\frac{\delta \omega}{2}} C(\omega) \mathrm{d} \omega .
\end{aligned}
$$

Obviously, $C(\omega)$ is the Fourier transform of $C(t)$ and $\bar{C}(\omega)$ a slightly "smeared out" version of it. Let $\bar{C}(t)$ be the back transform of $\bar{C}(\omega)$. Now, as long as $t \ll 2 \pi / \delta \omega$, one has $\bar{C}(t) \approx C(t)$. Thus

$$
\int_{0}^{\tau_{0}} C(t) \mathrm{d} t \approx \int_{0}^{\tau_{0}} \bar{C}(t) \mathrm{d} t
$$

as long as $\tau_{0} \ll 2 \pi / \delta \omega$. Since $\bar{C}(t)$ (other than $C(t)$ ) does not feature any recurrence time if $\bar{C}(\omega)$ is a smooth function of frequency, we may now drive the upper boundary of the second integral to infinity without much changing its value. This yields

$$
\int_{0}^{\tau_{0}} \bar{C}(t) \mathrm{d} t \approx \int_{0}^{\infty} \bar{C}(t) \mathrm{d} t=\pi \bar{C}(\omega=0) .
$$

(In the following we denote $\bar{C}(\omega=0)$ simply as $\bar{C}(0)$.) This, however, can only hold if $\tau_{c} \ll 2 \pi / \delta \omega$. A rough estimation for $\tau_{c}$ is given by $\tau_{c} \approx 2 \pi / \Delta \omega$ where $\Delta \omega$ is the width of the spectrum of $C(\omega)$. Consequently, $\Delta \omega \gg \delta \omega$ has to hold. Of course, $\delta \omega$ can always be chosen to fulfill this, but if it is chosen too small, $\bar{C}(\omega)$ is not necessary a smooth function of frequency. Thus (23) eventually holds if $\bar{C}(\omega)$ has a reasonably well defined "peak density" on a frequency scale small compared to its width $\Delta \omega$. In this case $\bar{C}(0)$ does not depend much on $\delta \omega$ and there is no need to define $\delta \omega$ with extreme precision.

The KF (also evaluated for a reduced two-subunit system with $V$, as routinely done, replaced by the number of contacts between identical subunits, i.e., $V=1$ ) and its limit for $\omega \ll T, L^{\prime}(\omega)$, read in terms of the correlation function

$$
L(\omega)=\pi \frac{1-\mathrm{e}^{-\omega / T}}{T \omega} C(\omega), \quad L^{\prime}(\omega)=\frac{\pi}{T^{2}} C(\omega) .
$$

This is to be compared with the conductivity as obtained from (19), 22), (23) which reads

$$
\kappa=\frac{\pi}{T^{2}} \bar{C}(0) .
$$

Obviously, $\kappa$ formally equals $\bar{L}(0)$, the peak density of $L(\omega)$ at frequency zero. As mentioned in Sect. II this quantity has been suggested to compute the conductivity of infinite systems from finite models. In this sense and for $t<\tau_{d}$ the $\mathrm{KF}$ is valid for the computation of thermal conductivity, although there is no external potential in this scenario. Note that the correlation function in the case of the KF is given by the full Heisenberg dynamics of the system based on the full Hamiltonian, whereas $\kappa$ is determined by the correlation function based on the interaction picture dynamics, i.e., without taking the interaction into account. However, since the whole theory is formulated for the weak coupling limit, this is not going to make a big difference as will be numerically demonstrated below (cf. Sect. VII).

So far we have always assumed that $\tau_{c}<\tau_{d}$. But is that justified? The first order term of the Dyson series is $\mathrm{i} \hat{U}_{1}$. Thus an estimate for the magnitude of the first order term which puts weight to the energy subspaces being proportional to their occupation probability is given by

$$
F(t):=\operatorname{Tr}\left\{\hat{\rho}_{0} \hat{U}_{1}^{2}\right\}
$$

Exploiting (12) one finds

$$
\frac{\mathrm{d}}{\mathrm{d} t} F(t) \approx \int_{0}^{t} \underbrace{\operatorname{Tr}\left\{\hat{\rho}_{0} \hat{V}(0) \hat{V}\left(t^{\prime}\right)\right\}}_{=: C_{V}\left(t^{\prime}\right)} \mathrm{d} t^{\prime} .
$$

Since the decay of the interaction auto-correlation $C_{V}\left(t^{\prime}\right)$ will roughly proceed on the same timescale as the current auto-correlation, the temporal change of $F(t)$ will assume a steady value after $\tau_{c}$. Thus, after $\tau_{c}$, one has $F(t) \approx$ $\bar{C}_{V}(0) t$, where $\bar{C}_{V}(0)$ is defined analogous to $\bar{C}(0)$ but based on the interaction auto-correlation. The time for a valid approximation according to the Dyson series may now be defined as

$$
\tau_{d}=\frac{1}{\bar{C}_{V}(0)},
$$

a time for which $F\left(\tau_{d}\right)=1$ and consequently the second order approximation definitely brakes down.

If, based on this definition, $\tau_{c}<\tau_{d}$ is not fulfilled the current cannot be shown to assume a steady value proportional to $\Delta T$ and thus Fourier's law will, in general, not be fulfilled. That means the model does not show normal transport. According to (27) this happens if the coupling becomes to strong. This fact might be missed by simply evaluating the KF: Since $C(\omega)$ completely scales with the square of the overall interaction strength (at least for $C(\omega)$ evaluated in the interaction picture), the distinction between normal and non-normal transport cannot be made by simply looking at the features of $C(\omega)$ (this will be demonstrated in more detail in Sect. VII). The transport behavior can also become non-normal if the coupling becomes too weak. Namely, for $\tau_{d} \gg 2 \pi / \delta \omega$ the current is well predicted by the truncated Dyson dynamics for times for which $\bar{C}(t) \approx C(t)$ does not hold any longer. This also means that the current cannot be predicted in the described way and thus Fourier's law will typically not be fulfilled (this will also be demonstrated in more detail in Sect. (VII). 


\section{HILBERT SPACE AVERAGE METHOD AND ITERATION SCHEME}

So far we have shown that, under given conditions on the model, for a short time period an energy current will flow between the subsystems which is proportional to the local temperature difference $\Delta T$. But this has been entirely derived under the assumption that the initial state was a local equilibrium state of the type $\hat{\rho}(T, \Delta T)$. So what happens after $\tau_{d}$ ? It is tempting to iterate the procedure, assuming that the state after $\tau_{d}$ would be again a local equilibrium state only with some reduced $\Delta T$. Unfortunately, this is rigorously not the case. At $t=0$ the initial state $\hat{\rho}(T, \Delta T)$ factorizes. This will for $t>\tau_{d}$ no longer be the case. The state at $t>\tau_{d}$ features an instantaneous current, $\hat{\rho}(T, \Delta T)$ does not. The full system's von-Neumann entropy always equals its initial entropy, but a local equilibrium state with reduced $\Delta T$ would feature a higher entropy.

Thus, in the following we abandon the local equilibrium state. Instead we show, that essentially almost all states that feature a certain $S$ induce, for a short timestep, a current that is proportional to $S$. This, of course, results in a state featuring an accordingly reduced $S$. Assuming that this state also belongs to the above class, the above short time-step dynamics for $S$ may be iterated. This yields continuous dynamics for $S$ and hence for the current. The assumption is only reliable if the above class contains basically all states in quest.

The formalism to implement this scheme is called Hilbert space average method (HAM). The key idea of this method is to replace the expectation values for observables $\hat{A}$ of actual pure states $\langle\psi|\hat{A}| \psi\rangle$ (in our case the expectation value of the energy skewness $\langle\psi|\hat{S}| \psi\rangle)$ by their Hilbert space averages

$$
\llbracket\langle\psi|\hat{A}| \psi\rangle \rrbracket_{\{\langle\psi|\hat{B}| \psi\rangle=b\}} .
$$

This expression stands for the average of $\langle\psi|\hat{A}| \psi\rangle$ over all $|\psi\rangle$ that feature $\langle\psi|\hat{B}| \psi\rangle=b$ but are uniformly distributed otherwise. Uniformly distributed means invariant with respect to all unitary transformations that leave $\langle\psi|\hat{B}| \psi\rangle=b$ unchanged. The replacement of actual expectation values by their Hilbert space averages is only a justified guess if almost all individual $|\psi\rangle$ yield expectation values close to the Hilbert space average of the observable. It can be shown that this is the case if the spectral width of $\hat{A}$ is not too large and $\hat{A}$ is high-dimensional. Full explanation of HAM is beyond the scope of this text and can be found in Ref ${ }^{24.26}$. Here HAM is only to be applied.

For the moment let the system be in a pure state. Then the current is given by the temporal change of $\langle\psi|\hat{S}(\tau)| \psi\rangle$. Assume that the following set of expectation values is known: $\langle\psi|\hat{P}(\eta)| \psi\rangle=P(\eta)$, where $\hat{P}(\eta)$ is a projector, projecting out the subspace which corresponds to an energy interval of width $\Delta \eta$ around $E=\eta$, i.e.,

$$
\hat{P}(\eta)=\sum_{E=\eta-\Delta \eta / 2}^{\eta+\Delta \eta / 2} \sum_{s}|E, s\rangle\langle E, s|,
$$

where $E$ are eigenvalues of $\hat{E}:=\hat{h}(1)+\hat{h}(2)$ (sum of local energies) and $s$ eigenvalues of $\hat{S}$ (energy skewness). Since, for high enough temperatures, the sum of the local energies is an approximately conserved quantity, the $P(\eta)$ are, for large enough $\Delta \eta$, also approximately conserved. Assume furthermore that the initial energy skewnesses within all subspaces $\eta$, i.e., $\langle\psi|\hat{P}(\eta) \hat{S}| \psi\rangle=S(\eta)$ are also known. Without taking any further information on $|\psi\rangle$ into account the best guess on the evolution of $\langle\psi|\hat{S}(\tau)| \psi\rangle$ is given by the Hilbert space average

$$
\llbracket\langle\psi|\hat{S}(\tau)| \psi\rangle \rrbracket_{\{\langle\psi|\hat{P}(\eta)| \psi\rangle=P(\eta),\langle\psi|\hat{P}(\eta) \hat{S}| \psi\rangle=S(\eta)\}}=\operatorname{Tr}\{\hat{S} \hat{\alpha}\}
$$

with

$$
\hat{\alpha}=\llbracket|\psi\rangle\langle\psi| \rrbracket_{\{\langle\psi|\hat{P}(\eta)| \psi\rangle=P(\eta),\langle\psi|\hat{P}(\eta) \hat{S}| \psi\rangle=S(\eta)\}} .
$$

Now what is the above Hilbert space average $\hat{\alpha}$ ? Any unitary transformation $\hat{G}$ that leaves $\hat{P}(\eta)$ and $\hat{P}(\eta) \hat{S}$ invariant has to leave $\hat{\alpha}$ invariant, i.e.,

$$
\begin{aligned}
& \mathrm{e}^{\mathrm{i} \hat{G}} \hat{\alpha} \mathrm{e}^{-\mathrm{i} \hat{G}}=\hat{\alpha} \quad \text { with } \\
& {[\hat{G}, \hat{P}(\eta) \hat{S}]=[\hat{G}, \hat{P}(\eta)]=0 .}
\end{aligned}
$$

This, however, can only be fulfilled if $[\hat{G}, \hat{\alpha}]=0$ for any $\hat{G}$. Furthermore, since the Hilbert space average $\hat{\alpha}$ is to be computed under some restrictions (see (32)), one has the following conditions

$$
\operatorname{Tr}\{\hat{\alpha} \hat{P}(\eta)\}=P(\eta), \quad \operatorname{Tr}\{\hat{\alpha} \hat{P}(\eta) \hat{S}\}=S(\eta) .
$$

According to the invariance properties of $\hat{\alpha}$ and the properties of the operators $\hat{P}(\eta)$ and $\hat{P}(\eta) \hat{S}(\underline{33})$, one may thus write $\hat{\alpha}$ as

$$
\hat{\alpha}=\sum_{\eta}\left(P(\eta) \frac{\hat{P}(\eta)}{\operatorname{Tr}\{\hat{P}(\eta)\}}+S(\eta) \frac{\hat{P}(\eta) \hat{S}}{\operatorname{Tr}\left\{\hat{P}(\eta) \hat{S}^{2}\right\}}\right) .
$$

Defining $\hat{\rho}_{0}(\eta):=P(\eta) \hat{P}(\eta) / \operatorname{Tr}\{\hat{P}(\eta)\}$, (35) may be rewritten as

$$
\hat{\alpha}=\sum_{\eta} \hat{\rho}_{0}(\eta)\left(\hat{1}+\frac{S(\eta)}{\operatorname{Tr}\left\{\hat{\rho}_{0}(\eta) \hat{S}^{2}\right\}} \hat{S}\right) .
$$

By choosing $P(\eta)$ and $S(\eta)$ to be equal to the expectation values of $\hat{\rho}(T, \Delta T)$ for the respective operators, i.e.,

$$
\begin{aligned}
& P(\eta):=\operatorname{Tr}\{\hat{P}(\eta) \hat{\rho}(T, \Delta T)\}=\operatorname{Tr}\left\{\hat{\rho}_{0} \hat{P}(\eta)\right\} \\
& S(\eta):=\operatorname{Tr}\{\hat{P}(\eta) \hat{S} \hat{\rho}(T, \Delta T)\}=\frac{\Delta T}{T^{2}} \operatorname{Tr}\left\{\hat{\rho}_{0}(\eta) \hat{S}^{2}\right\}
\end{aligned}
$$

one gets $\sum_{\eta} \hat{\rho}_{0}(\eta) \approx \hat{\rho}_{0}$ and thus $\hat{\alpha} \approx \hat{\rho}(T, \Delta T)$ as can be seen from comparison with (9). Hence the Hilbert space 
average over all pure states featuring the same expectation values for $\hat{P}(\eta)$ and $\hat{P}(\eta) \hat{S}$ as $\hat{\rho}(T, \Delta T)$ is $\hat{\rho}(T, \Delta T)$ itself. Therefore

$$
\langle\psi|\hat{S}(\tau)| \psi\rangle \approx \operatorname{Tr}\{\hat{\rho}(T, \Delta T) \hat{S}(\tau)\}
$$

i.e., any pure state (regardless of whether it is entangled with respect to the subunits or whether its local entropy is maximum) featuring the same expectation values for $\hat{P}(\eta)$ and $\hat{P}(\eta) \hat{S}$ as the local equilibrium state $\hat{\rho}(T, \Delta T)$ is most likely to yield the same local current as the local equilibrium state. Since incoherent mixtures of pure states featuring the same expectation values for $\hat{P}(\eta)$ and $\hat{P}(\eta) \hat{S}$ as the local equilibrium state are even closer to the latter then pure states, they are even more likely to induce the same current as the local equilibrium state. This holds for the set of $P(\eta), S(\eta)$ as given by (37). But how will this set look like after $\tau_{0}$, i.e., how do the $P(\eta)$ and $S(\eta)$ evolve during the time $\tau_{0}$ ? Since the $P(\eta)$ are approximate constants of motion, they remain invariant. For the $S(\eta)$ we find from applying the scheme developed in Sect. $\nabla$ to $\operatorname{Tr}\{\hat{\alpha} \hat{P}(\eta) \hat{S}(\tau)\}$ [cf. (18), (19), (25), (37)]

$$
S\left(\eta, t+\tau_{0}\right) \approx S(\eta, t)-\underbrace{\frac{\pi \bar{C}(\eta, 0)}{\operatorname{Tr}\left\{\hat{\rho}_{0}(\eta) \hat{S}^{2}\right\}}}_{=: 2 D(\eta)} S(\eta, t) \tau_{0},
$$

where $\bar{C}(\eta, 0)$ is analogous to $\bar{C}(0)$ as defined by (20) and (18) but $\hat{\rho}_{0}$ replaced by $\hat{\rho}_{0}(\eta)$. If the criteria from Sect. $\nabla$ are fulfilled and $\tau_{0}$ is comparatively short, this may now be iterated yielding

$$
\frac{\mathrm{d}}{\mathrm{d} t} S(\eta)=-2 D(\eta) S(\eta)
$$

Thus, in general, any energy subspace has its own energy diffusion coefficient. Therefore it might, be impossible to describe the energy diffusion behavior between the subunits entirely by one overall conductivity as implied by the KF, even if all the criteria from (18) are fulfilled. However, if the $D(\eta)$ corresponding to the energy subspaces that are occupied with significant weight and contribute significantly to the transport feature similar values, i.e, $D(\eta) \approx D$, one gets only one single diffusion coefficient that then reads

$$
D \approx \frac{\pi \bar{C}(0)}{\operatorname{Tr}\left\{\hat{\rho}_{0} \hat{S}^{2}\right\}} .
$$

(Note that $\left.\sum_{\eta} \bar{C}(\eta, 0)=\bar{C}(0)\right)$. In this case one might sum (40) over $\eta$ which yields

$$
\frac{\mathrm{d}}{\mathrm{d} t} S=-2 D S
$$

It is straightforward to show that the heat capacity $c$ for one subsystem is given by $c=2 \operatorname{Tr}\left\{\hat{\rho}_{0} \hat{S}^{2}\right\} / T^{2}$. Exploiting this and (41) one may rewrite (42) as

$$
\frac{\mathrm{d}}{\mathrm{d} t} S=-\frac{2 \kappa}{c} S \quad \text { or } \quad j(1)=\frac{\kappa}{c}(h(1)-h(2))
$$

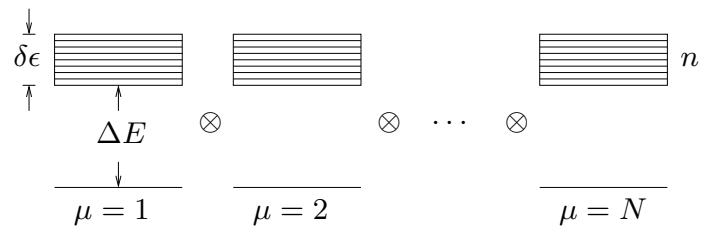

FIG. 1: Simple model to analyze diffusive energy transport: $N$ coupled subunits featuring a single ground state and an excitation band of $n$ equally distributed levels.

which, generalized to $N$ subsystems, identifying $D=\kappa / c$ and combined with the discrete continuity eq., yields (5). Hence this closes the loop for a microscopic derivation of (5). This essentially means that if the criteria for $\tau_{0}, \tau_{c}, \tau_{d}$ from Sect. $\nabla$ are fulfilled and the relevant $D(\eta)$ are similar, energy will diffuse from subunit to subunit as predicted by Fourier's law.

\section{APPLICATION TO MODELS}

In this Section the previously derived theoretical results are compared with numerical data in the following way: For concretely defined models the energy diffusion coefficient $D=\kappa / c$ which appears in (5) is computed from the KF. Then (5) is solved for some nonuniform initial energy distribution. Furthermore, the time-dependent Schrödinger eq. is solved numerically for an initial state corresponding to the above initial energy distribution. From those data the exact evolution of the local energies $h(\mu)=\langle\psi(t)|\hat{h}(\mu)| \psi(t)\rangle$ is computed and compared with the above solution of (5). Only if there is good agreement, the system exhibits normal transport that may be characterized by the conductivity obtained from the KF.

We introduce two classes of models which are primarily designed to represent modular systems in general rather than real physical systems. (For the impact on real physical systems see Sect. III) The first class of models features subunits with non-degenerate ground states, large energy gaps $(\Delta E)$ and one, comparatively narrow energy band $(\delta \epsilon)$ each, as depicted in Fig. 11 Within one band there are $n$ states featuring equidistant level spacing. The next neighbor interactions are defined as

$$
\hat{V}=\lambda \sum_{i, j, \mu} v(i, j) \hat{P}^{+}(i, \mu) \hat{P}^{-}(j, \mu+1)+\text { h.c. }
$$

(h.c. stands for the hermitian conjugate of the previous sum.) Here $\hat{P}^{+}(i, \mu)$ corresponds to a transition of the $\mu$ 'th subunit from its ground state to the $i$ 'th eigenstate of the band. $\hat{P}^{-}$corresponds to the respective downwards transition. The $v(i, j)$ are randomly distributed complex numbers normalized to $\sum_{i, j}|v(i, j)|^{2} / n^{2}=1$. Thus $\lambda$ sets the overall interaction strength. Due to this model design only one energy subspace contributes to transport at all, which is the "one-excitation subspace" defined by 


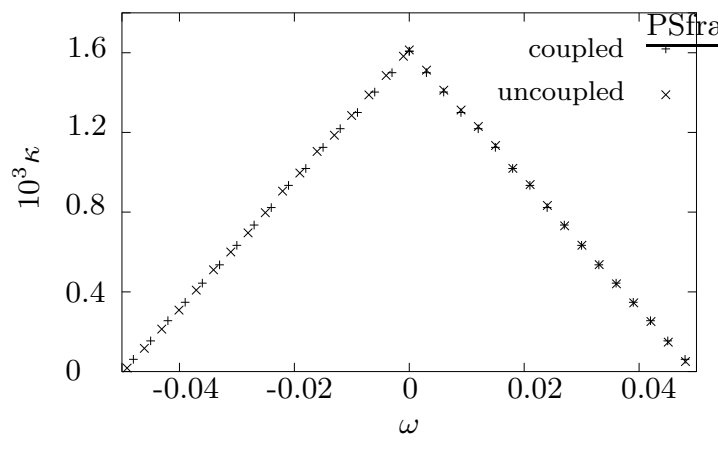

FIG. 2: Heat conductivity over frequency for a ringlike system as depicted in Fig. 1 $(N=6$, other parameters see text). Crosses refer to the coupled system, as intended by the Kubo formula, and x's to the decoupled system, as suggested in Sect. D

an overall energy $E$ with $\Delta E \leq E \leq \Delta E+\delta \epsilon$. The dimension $M=n N$ of this relevant subspace grows linearly rather than exponentially with the number of subunits. This allows to numerically analyze models with high enough $n$ to fulfill all criteria for diffusive transport, but also up to fifteen subunits. Thus those models are meant to check whether or not the locally computed conductivity holds for arbitrarily many subunits, as predicted by the theory. (For a "stand alone" treatment of such systems, cf. Ref ${ }^{27}$.)

We consider a ring of $N=6$ subsystems with $n=500$, $\lambda=5 \cdot 10^{-5}, \Delta E=10$ and $\delta \epsilon=0.05$. We find that with a frequency averaging interval of $\delta \omega \approx 10^{-3}=\delta \epsilon / 50$ all the conditions mentioned in Sect. $\mathbb{\nabla}$ are fulfilled. Since only one energy subspace contributes to transport, none of the difficulties concerning different $L(\eta)$ discussed in Sect. [V] arises. Thus the KF may be evaluated at any temperature to compute $D=\kappa / c$. We evaluated $\kappa(\omega)=\bar{C}(\omega) / T^{2}$ (cf. Sect. $\mathbb{V}$ ) for $T=1.4$ on the basis of the coupled system, as intended by the Kubo formula, and on the basis of the uncoupled system, as intended by our argument in Sect. $\nabla$ Both results are displayed in Fig. 2. Obviously, there is a good agreement between the two graphs, it appears to be irrelevant whether the correlation function is evaluated on the basis of the coupled or the decoupled system. (It should be mentioned that in both cases $C(\omega)$ features finite contributions exactly at $\omega=0$, thus there is a finite Drude peak.) From Fig. 2 we find $\kappa=1.6 \cdot 10^{-3}(\omega=0)$ and calculating the specific heat for one subunit yields $c(T=1.4)=10.5$. This yields $D=3.142 \cdot 10^{-4}$. The corresponding solution of (15) for all energy initially concentrated in one subsystem is shown in Fig. 3] Furthermore, we solved the Schrödinger eq. for a corresponding pure initial product state, featuring one subunit in a randomly generated state restricted to the excitation band, and all other subunits in their ground states. The result is also shown in Fig. [3 Obviously, there is fairly good agreement. We checked rings up to fifteen subunits and always found good agreement. So far the KF appears to be perfectly valid for heat conduction.

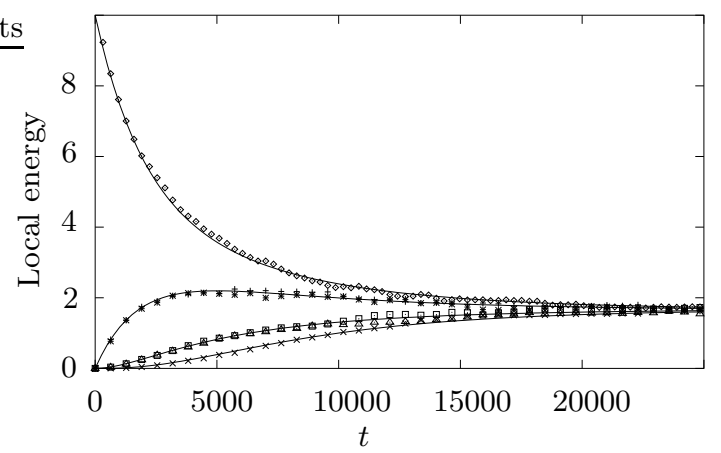

FIG. 3: Evolution of the local energies of a weakly coupled system as depicted in Fig. 11 The initial state features one excited subsystem. Displayed are the predictions from the Kubo formula (solid lines) and the complete exact solution of the Schrödinger equation (points). The figure indicates diffusive transport in accord with the Kubo formula.

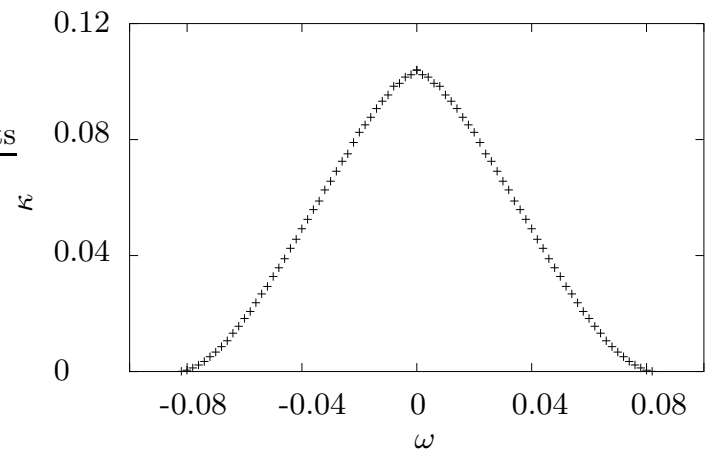

FIG. 4: Conductivity over frequency as calculated from the Kubo formula for a strongly coupled system as depicted in Fig. 1

But are the criteria for diffusive transport from Sect. $\mathbb{V}$ fulfilled? An estimate for the correlation time is given by $\tau_{c} \approx 2 \pi / \delta \epsilon \approx 10^{2}$. From evaluating (28) one finds $\tau_{d} \approx 7 \cdot 10^{3}$. Thus $\tau_{c} \ll \tau_{d}$ obviously holds. Furthermore $2 \pi / \delta \omega \approx 6 \cdot 10^{3}$, hence $\tau_{d} \approx 2 \pi / \delta \omega$, i.e., the criteria for diffusive transport are fulfilled.

If, however, the interaction strength is such that the criteria from Sect. $\nabla$ are not fulfilled the transport behavior ceases to be diffusive, i.e., the good agreement between the solution of (5) and the solution of the Schrödinger eq. vanishes. For example, for a model like the above one but with $\lambda=5 \cdot 10^{-4}$ one has $\tau_{d} \approx 70$ and $\tau_{c} \ll \tau_{d}$ is not fulfilled. Fig. 5 shows the significant deviations of the evolution of the local energies from normal diffusive behavior as described by (5). But the graph for $\kappa(\omega)$ as calculated from the Kubo formula (see Fig. (4) does not look essentially different from the above regular case. In particular there is no pronounced singularity at $\omega=0$ as expected for the case of ballistic transport. Thus it is not obvious how the general transport behavior is to be found from simply evaluating the Kubo formula since there is no $\tau_{d}$ to be checked. The same is found for extremely weak interactions. For example, for a model like 


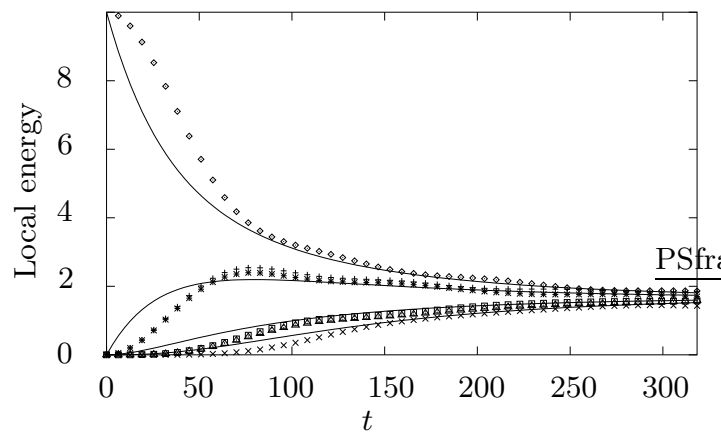

FIG. 5: Evolution of the local energies of a strongly coupled system as depicted in Fig. 11 The deviation of the points (Schrödinger equation) from the solid lines (Fourier's law, Kubo formula) indicate the breakdown of diffusive behavior and the validity of the Kubo Formula.

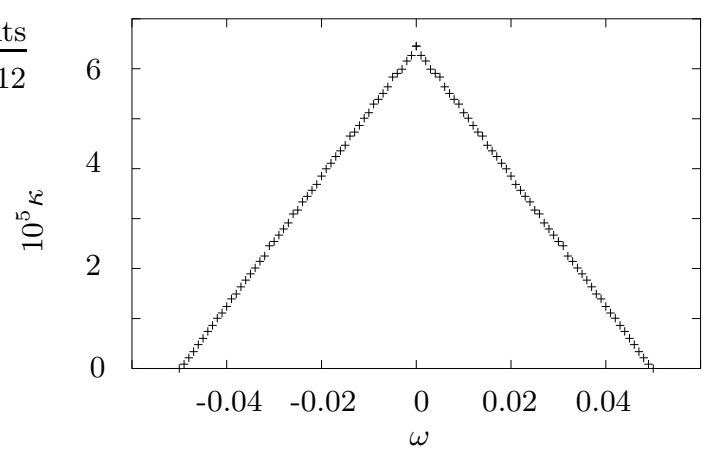

FIG. 6: Conductivity over frequency as calculated from the Kubo formula for an extremely weak coupled system as depicted in Fig. 1

the above one but with $\lambda=10^{-5}$ one has $\tau_{d} \approx 1.8 \cdot 10^{5}$ and $\tau_{d} \approx<2 \pi / \delta \omega$ is not fulfilled. Consequently, the regular transport behavior breaks down (see Fig. 7). But again, the graph for $\kappa(\omega)$ as calculated from the Kubo formula does not look essentially different from the regular case (see Fig. [6).

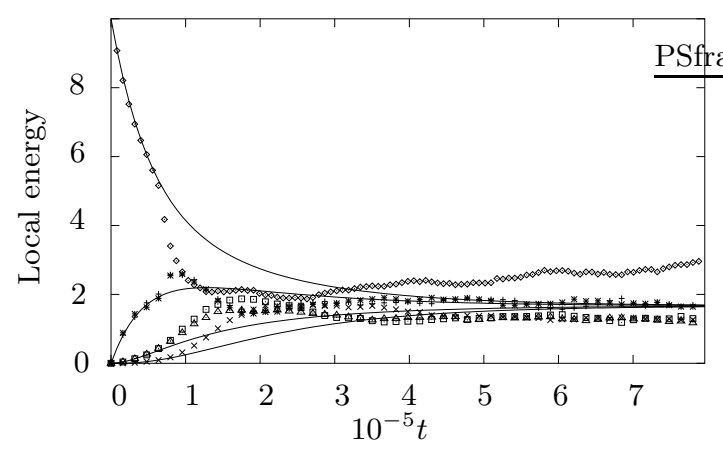

FIG. 7: Evolution of the local energies of an extremely weakly coupled system as depicted in Fig. 1 The deviation of the points (Schrödinger equation) from the solid lines (Fourier's law, Kubo formula) indicate the breakdown of diffusive behavior and the validity of the Kubo Formula.

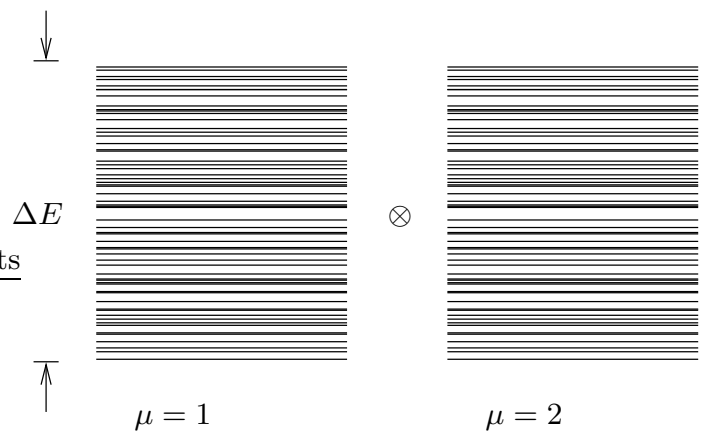

FIG. 8: Gapless model to analyze diffusive transport: Two coupled subunits featuring $n$ levels which are uniformly distributed within the energy interval $\Delta E$.

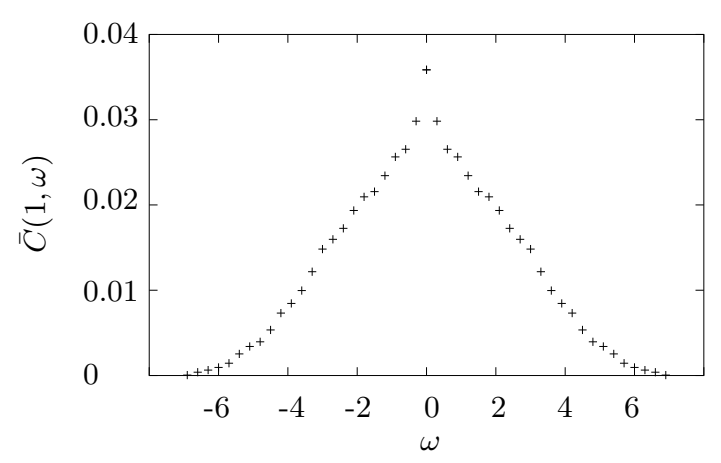

FIG. 9: Fourier transform of the current auto-correlation function for the model depicted in Fig. 8 (parameters see text).

The other model is meant to show that the gapped spectrum of the subunits is dispensable for demonstrating diffusive transport. It consists of subunits featuring $n$ eigenstates distributed uniformly within an energy interval $\Delta E$ as depicted in Fig. 8 Here the interaction is chosen to be a complex random matrix on the full system's space without any restriction to a subspace. Neverthe-

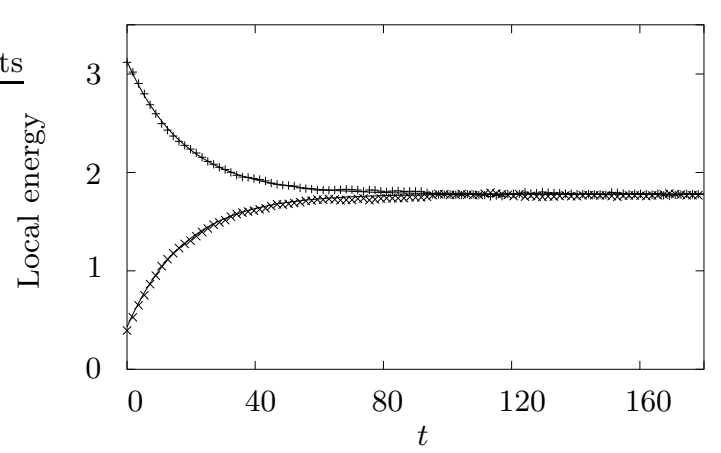

FIG. 10: Evolution of the local energies for the model depicted in Fig. 8 for an initial state featuring $T_{1} \gg \Delta E, T_{2} \ll \Delta E$. Solid lines refer to the HAM prediction (5), points to the Schrödinger equation. The model clearly shows diffusive transport. 
less, the interaction is supposed to be given by $\hat{V}=\lambda \hat{v}$ with $\operatorname{Tr}\left\{\hat{v}^{2}\right\} / n^{2}=1$. To keep the problem numerically manageable we consider only two subunits. We specify our model concretely by $N=2, n=60, \lambda=5 \cdot 10^{-3}$, $\Delta E=7$. For this case we consider a ("pseudo-thermal") pure product initial state. The amplitudes of this state are chosen such that their magnitude squares obey Boltzmann distributions with $T_{1}=40, T_{2}=1$. The phases of the amplitudes are chosen at random. This state has been chosen since for states with lower temperature differences it is hard to distinguish relaxation behavior from fluctuations. Since for this model various energy subspaces $\eta$ yield different transport types and coefficients $D(\eta)$, the transport behavior for this initial state cannot simply be determined by directly applying the Kubo formula. It may, however, be analyzed within the framework described in Sect.VI. Since $\Delta \eta$ eventually determines the correlation time $\tau_{c}$ corresponding to the energy subspace $\eta$, one must, as eventually turns out, divide the energy scheme of this model in just two subspaces. Thus $\hat{P}(1)$ projects out all states with total energy $E$ lower than $\Delta E$, $0 \leq E \leq \Delta E$, and $\hat{P}(2)$ the states with $\Delta E<E \leq 2 \Delta E$. Based on those definitions one finds for the above initial state $P(1) \approx 1, P(2) \approx 0$. Thus the relaxation behavior is controlled by $D(1)$ as given by (39). Fig. 9] shows the corresponding $\bar{C}(1, \omega)$ and we find $\bar{C}(1,0)=0.036$. According to the above definitions we have $\tau_{c} \approx 2 \pi / \Delta E \approx 0.9$. From numerically evaluating $\tau_{d}$ we find $\tau_{d} \approx 40$ and thus the corresponding condition for normal energy diffusion $\tau_{c} \ll \tau_{d}$ is fulfilled. With $\delta \omega=0.3$ we get $2 \pi / \delta \omega \approx 21$ which is of the same order of magnitude as $\tau_{d}$, which also indicates diffusive transport. From numerical evaluation we furthermore find $\operatorname{Tr}\left\{\hat{\rho}_{0}(1) \hat{S}^{2}\right\}=2.03$. Those numbers eventually yield $D(1)=0.0139$. Indeed, as displayed in Fig. 10, there is good agreement of the solution of (5) based on this $D(1)$ with the full numerical solution of the corresponding Schrödinger equation.

\section{SUMMARY AND CONCLUSION}

We investigated the energy transport behavior of modular quantum systems, i.e., systems that can be described as weakly coupled identical many-level subunits. We argued that this description may apply to a large class of systems. For simplicity, we concretely analyzed modular chains and rings with next neighbor couplings. Without making any reference to external forces or exploiting the hypothesis of local equilibrium we showed that those systems may or may not exhibit normal heat transport, but if they do, the conductivity is correctly described by the Kubo formula. This is in accord with Refs, 13.17.18 where the Kubo Formula has been evaluated for concrete systems and the results have been counter-checked by either experiments or other theoretical methods. This is furthermore in accord with Refs 28.29 .30 .31 .32 where it is shown that one-dimensional systems may or may not exhibit diffusive heat transport.

We also suggested general criteria to decide whether or not such modular systems exhibit normal transport. Those criteria are established on the basis of the concrete form of the subunits and their mutual interactions. We found, however, that the question cannot be decided only by evaluating the Kubo Formula. To check our theoretical results we introduced some examples for concrete, finite, modular, chainlike systems. For those examples we numerically solved the time-dependent Schrödinger equation which yields the energy transport dynamics. Those dynamics are in accord with our above mentioned theoretical results.

The results support the view that thermodynamic behavior might, under specific conditions on the system, emerge directly from quantum mechanics 24.33 .34 . These conditions do not necessarily include a many particle limit.

We sincerely thank F. Heidrich-Meisner for very fruitful discussions. Financial support by the Deutsche Forschungsgesellschaft is gratefully acknowledged.
* Electronic address: jgemmer@uos.de

1 R. Kubo, J. Phys. Soc. Jpn. 12, 570 (1957).

${ }^{2}$ R. Kubo, M. Yokota, and S. Nakajima, J. Phys. Soc. Jpn. 12, 1203 (1957).

3 J. M. Luttinger, Phys. Rev. 135, A1505 (1964).

${ }^{4}$ R. Kubo, M. Toda, and N. Hashitsume, Statistical Physics II: Nonequilibrium Statistical Mechanics, no. 31 in SolidState Sciences (Springer, Berlin, Heidelberg, New-York, 1991), 2nd ed.

5 S. Lepri, R. Livi, and A. Politi, Physics Reports 377, 1 (2003).

${ }^{6}$ D. N. Zubarev, Nonequilibrium Statistical Thermodynam- ics, Studies in Soviet Sciences (Consultants Bureau, New York, London, 1974), transl. by P. J. Shepherd.

7 P. Glansdorff and I. Prigogine, Thermodynamics Theory of Structure, Stability and Fluctuations (Wiley, London, 1971).

8 G. D. Mahan, Many-Particle Physics (Plenum Press, New York, London, 2000), 3rd ed.

9 D. Forster, Hydrodynamic Fluctuations, Broken Symmetry, and Correlation Functions (W. A. Benjamin, Inc.; Advanced Book Program Reading, Massachusetts, London, 1975).

10 S. de Groot, Non-equilibrium thermodynamics (Wis- 
senschaftsverlag, Mannheim, Wien, Zürich, 1962).

11 P. Garrido, P. Hurtado, and B. Nadrowski, Phys. Rev. Lett. 86, 5486 (2001).

12 F. Bonetto, J. Lebowitz, and L. Rey-Bellet, Mathematical Physics 2000 (World Scientific Publishing Company, 2000), chap. Fourier's Law: A Challenge to Theorists, pp. $128-150$.

13 K. Saito and S. Miyashita, J. Phys. Soc. Jpn. 71, 2485 (2002).

14 M. Michel, M. Hartmann, J. Gemmer, and G. Mahler, Eur. Phys. J. B 34, 325 (2003).

15 M. Michel, J. Gemmer, and G. Mahler, Eur. Phys. J. B 42, 555 (2004).

16 M. Michel, J. Gemmer, and G. Mahler, Physica E 29, 129 (2005).

17 P. Allen and J. Feldman, Phys. Rev. B 48, 12581 (1993).

18 J. Feldman, M. Kluge, P. Allen, and F. Wooten, Phys. Rev. B 48, 12589 (1993).

19 X. Zotos, F. Naef, and P. Prelovsek, Phys. Rev. B 55, 11029 (1997).

20 F. Heidrich-Meisner, A. Honecker, D. Cabra, and W. Brenig, Phys. Rev. B 68, 134436 (2003).

21 A. Klümper and K. Sakai, J. Phys. A: Math. Gen. 35, 2173 (2002).

${ }^{22}$ F. Heidrich-Meisner, Ph.D. thesis, Technische Universität
Braunschweig, Braunschweig (2005).

${ }^{23}$ F. Heidrich-Meisnera, A. Honecker, D. Cabrab, and W. Brenig, Physica B 359-361, 1394 (2005).

24 J. Gemmer, M. Michel, and G. Mahler, Quantum Thermodynamics: Emergence of Thermodynamical Behavior within Composite Quantum Systems, LNP657 (Springer, Berlin, Heidelberg, New-York, 2004).

25 J. Gemmer and M. Michel, Physica E 29, 136 (2005).

26 J. Gemmer and G. Mahler, Eur. Phys. J. B 31, 249 (2003).

27 M. Michel, G. Mahler, and J. Gemmer, Phys. Rev. Lett. 95, 180602 (2005).

28 T. Prosen and D. Campbell, Phys. Rev. Lett. 84, 2857 (2000).

29 B. Li, G. Casati, J. Wang, and T. Prosen, Phys. Rev. Lett. 92, 254301 (2004).

${ }^{30}$ K. Saito, S. Takesue, and S. Miyashita, Phys. Rev. E 54, 2404 (1996).

31 K. Saito, Europhys. Lett. 61, 34 (2003).

32 C. Mejia-Monasterio, T. Prosen, and G. Casati, Eur. Phys. Lett. (2005), E-first, cond-mat/0504181

33 J. Gemmer, A. Otte, and G. Mahler, Phys. Rev. Lett. 86, 1927 (2001).

34 W. H. Zurek and J. P. Paz, Phys. Rev. Lett. 72, 2508 (1994). 